\title{
THE EFFECT OF SUPERPLASTICIZERS ON THE PROPERTIES OF GAMMA IRRADIATED CEMENT PASTES
}

\author{
"JAROSLAVA KOŤÁTKOVÁ*,**, ZBYNĚK HLAVÁČ*, VÍT ROSNECKÝ*, ROMAN MOHYLA*, JINDŘICH JANSA* \\ *Research Centre Rez, Hlavní 130, 250 68, Husinec- Řež, Czech Republic \\ ${ }^{* *}$ Czech Technical University in Prague, Faculty of Civil Engineering, Experimental Centre, \\ Thákurova 7, 166 26, Prague 6 Dejvice, Czech Republic \\ "E-mail: jaroslava.kotatkova@cvrez.cz
}

Submitted April 3, 2018; accepted May 22, 2018

\begin{abstract}
Keywords: Superplasticizer, Irradiated cement paste, Mechanical properties, Temperatures
Considering the safety criteria on radioactive waste disposal, all materials within the repositories usually have to meet very strict requirements. Low and intermediate level waste can be immobilised in cement composites for its well-proven properties, however there are gaps in the knowledge relating to the interaction of organic species, such as superplasticizers, with the cement paste and the irradiation stability of the final composite. The presented work studies the effects of gamma irradiation on the mechanical properties of cement pastes incorporating high amounts of two kinds of superplasticizers. In addition to this, temperature development due to the irradiation inside the pastes was studied in order to recognise potential temperature gradients. The obtained results showed insignificant changes of bulk density and scatter in values obtained by non-destructive methods. Destructive testing, however, revealed a positive effect of the irradiation on both the flexural and compressive strength of the studied pastes. The overall temperature changes were within $2^{\circ} \mathrm{C}$, having no significant effects on the properties of the pastes.
\end{abstract}

\section{INTRODUCTION}

The continuous production of radioactive waste, whether from the nuclear industry, agriculture, research facilities or medicine, calls for the safe and final disposal of the waste. Low and intermediate level waste can be stored in near-surface disposal sites (NEDS) or in deep geological repositories (DGR) in a separate part of the whole complex. Regardless of the place of its end point, the waste has to be immobilised within a solid durable material. Cement composites may be a suitable alternative to the more or less problematic matrices, such as geopolymers or bitumen, for its radiation and thermal stability, easy operation and time-proven properties [1,2]. The presence of organic substances is undesirable, especially in deep geological repositories (DGR), due to its fast degradation and alternation of redox conditions within the underground complex. Some organic substances (ligands) may form stable complexes decreasing the radionuclides uptake [3]. Immobilisation of radioactive waste in bitumen is, therefore, only a provisory solution, which cannot be stored in a DGR in any way. Moreover, cement matrices may contain organic substances in the form of additives, such as superplasticizers, setting accelerators, stabilisers, etc. Despite the fact, that these are present in very small amounts in the cement composites and their effects are expected to be negligible, it is necessary to approve precautions due to safety issues. The main aim of investigation must be the interaction of organic substances in cement paste with radioactive species and the stability against gamma irradiation, especially since the gained knowledge relating the topic is very low so far. The presented work focuses on the effects of a high dose of superplasticizers on the properties of gamma irradiated cement pastes.

\section{EXPERIMENTAL}

Cement pastes were manufactured with the use of Portland cement CEM I $42.5 \mathrm{R}$ and the water to cement ratio was 0.30 . Two different kinds of superplasticizers were selected and dosed in 10 times higher than the recommended dose in order to enhance the scale of the effects on the cement paste when irradiated. The used superplasticizers were: Melment F10, based on melamine formaldehyde resin; and a poly-carboxylate superplasticizer - Melflux 4930. The doses were 20\% of the cement weight for Melment F10 (pastes are marked as MF20) and $5 \%$ of cement weight for Melflux 4930 (marked as M5), the reference samples (CM_R) do not contain any plasticizer. The prepared pastes of dimensions $40 \times 40 \times 160 \mathrm{~mm}$ were cured in laboratory conditions. 


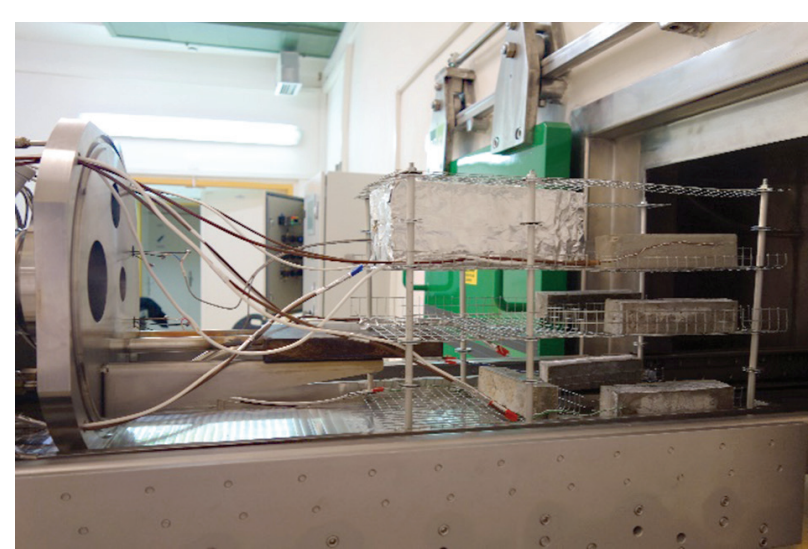

Figure 1. Samples prepared to be inserted into the irradiation chamber.

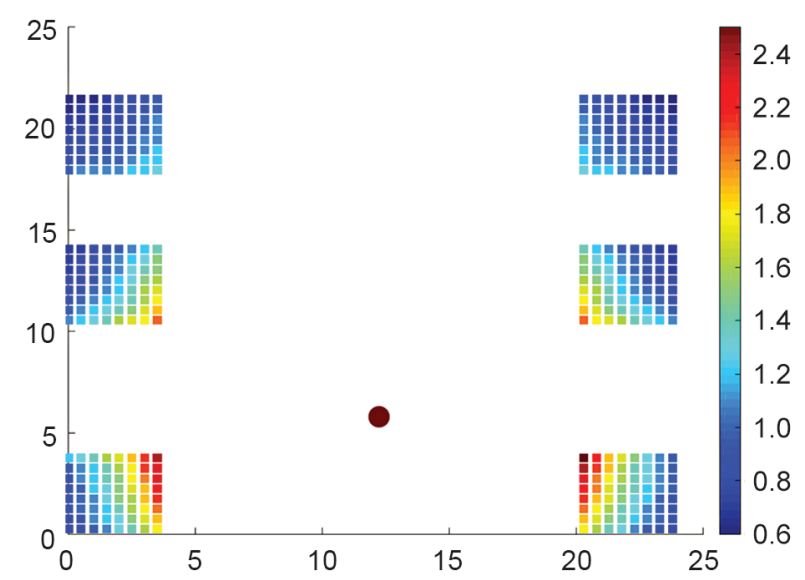

Figure 2. The distribution of radiation doses in the cross-sections of the samples.
At the age of 14 days, the pastes were tested non-destructively with the use of the ultrasound pulse velocity method to obtain the values of the dynamic modulus of elasticity $E_{c u}$ according to ČSN 731371 [4] and also, by using the impact echo method giving the dynamic modulus of elasticity in longitudinal direction $E_{c r}$, transversal direction $E_{c r f}$ and shear modulus $G_{c r}$ following the procedure of the Czech standard ČSN 73 1371 [6]. Half of the samples (3 samples for each type of cement paste) were placed in an irradiation chamber with a gamma source Co-60 and irradiated for $18+21$ days (Figure 1). The other set of samples was left in laboratory conditions to simply age.

The rate of irradiation was from 0.5 to $4.5 \mathrm{kGy}$ per hour and the cumulative obtained dose of samples ranged from 0.6 to $2.5 \times 10^{6} \mathrm{~Gy}$ according to the orientation of the samples towards the gamma source (Figure 2). During irradiation, the temperature development inside the pastes was measured at discrete time points to study the temperature increase in the samples caused by irradiation and its changes due to different modes of irradiation. One selected sample (M5-3) was insulated in a mineral insulation to observe the scale of the higher temperature increase.

After the end of the irradiation, all the samples were firstly non-destructively tested to obtain the modulus of elasticity and afterwards tested destructively to determine the flexural and compressive strength according to ČSN EN 1961 [5].

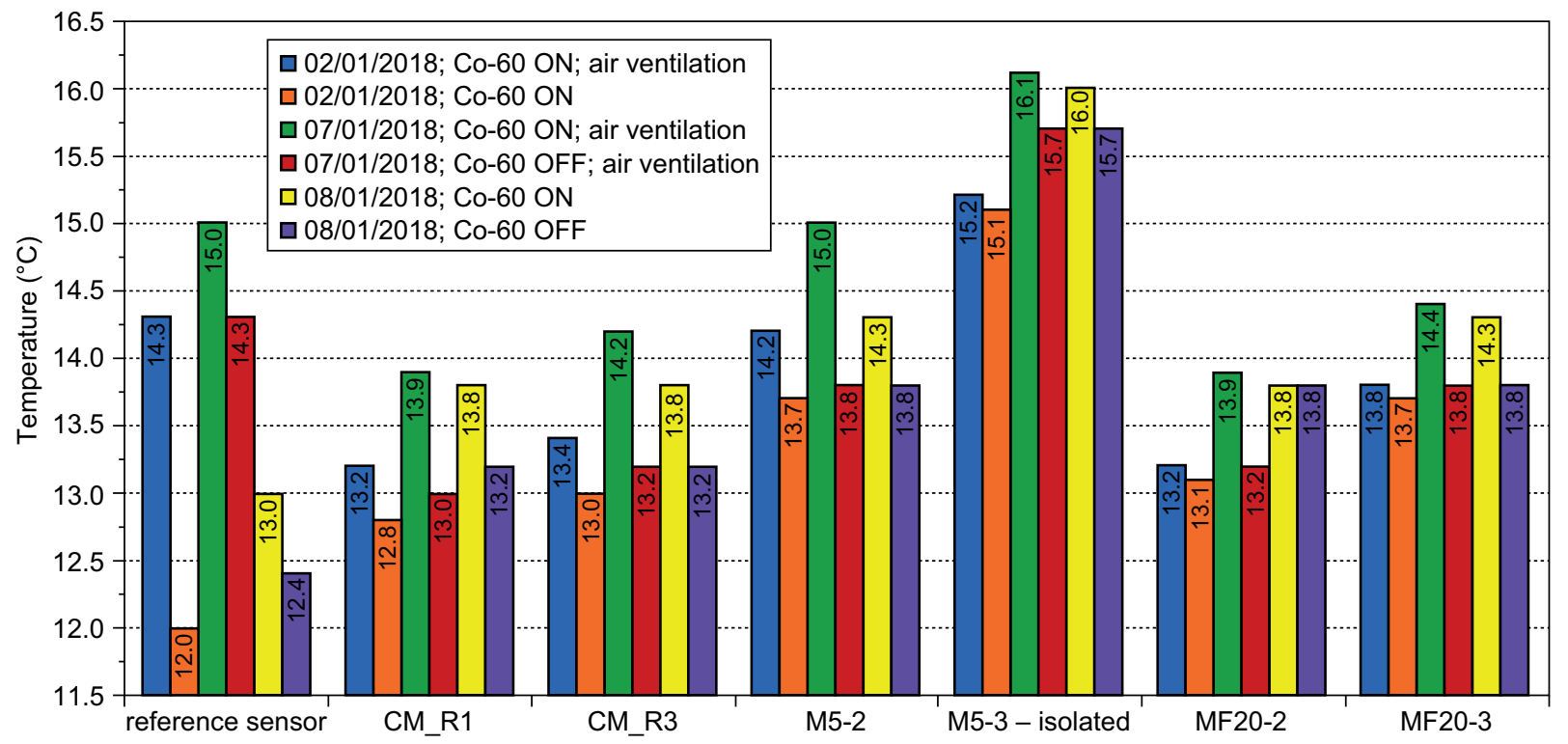

Figure 3. Temperature development inside the mass of the samples against the time and its response to changes in the irradiation mode (explanatory notes: Co-60 ON/OFF - the source of the irradiation in/out the chamber, i.e., the samples are/are not being irradiated; air ventilation - the ventilation mechanism is on) 


\section{RESULTS AND DISCUSSION}

Figure 3 presents the temperature development inside the cement pastes and its response to the different modes of irradiation. Thermocouples were inserted in two out of three irradiated samples of each paste type. From time to time, the air needed to be ventilated to get rid of the ionised air within the service room. It is evident that, when the air ventilation is on, the outside cold air is drawn in and, thus, the temperature in the service room decreases and, as a result, the temperatures inside the irradiation chamber increase by up to $2^{\circ} \mathrm{C}$. However, the difference inside the pastes caused by the ventilation is at most $1{ }^{\circ} \mathrm{C}$. The changes in temperature caused by the positioning of the gamma source are reflected in the differences of the columns marked as Co-60 ON and Co-60 OFF, meaning that the gamma source is positioned in the irradiation chamber and samples are being irradiated and the gamma source is out the chamber, i.e., the samples are not being irradiated, respectively. The changes are within $1^{\circ} \mathrm{C}$, and exceptionally by $1.2^{\circ} \mathrm{C}$ in the case of M5-2. There are bigger changes relating the position of the gamma source when the air ventilation is on. In the case of insulated sample M5-3, the absolute

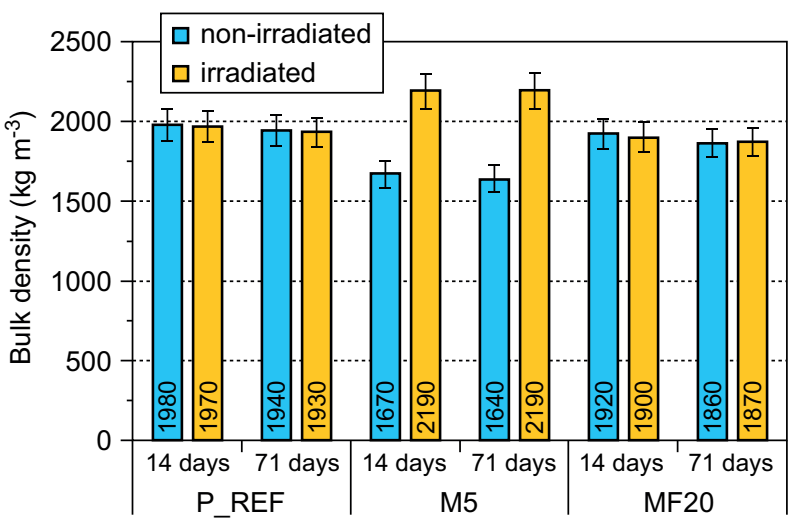

Figure 4. Bulk density of the samples determined before and after irradiation compared to the non-irradiated ones.

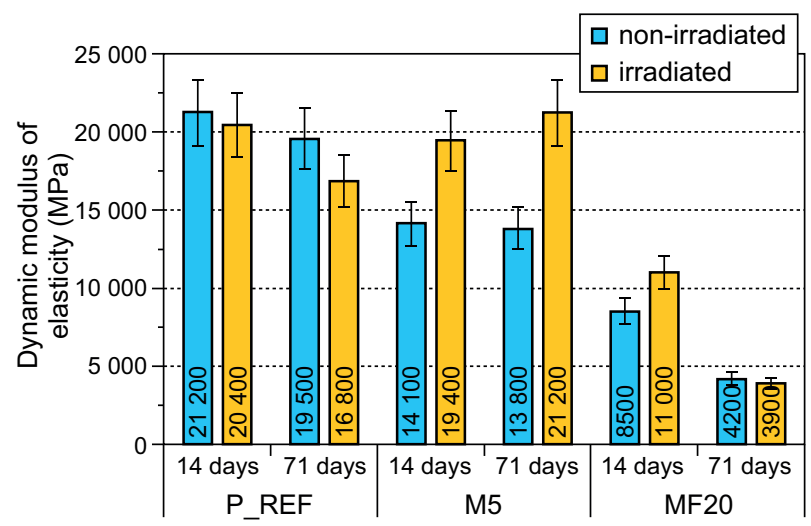

Figure 5. The dynamic modulus of elasticity measured by the ultrasound pulse velocity method before and after irradiation compared to the non-irradiated samples. temperatures are higher compared to the other specimen, while the relative changes in the temperature reflecting the mode of irradiation are slightly lower. To sum up, the changes in cement pastes within $2^{\circ} \mathrm{C}$ do not present any potential threat of a significant temperature gradient creation.

Changes in the bulk density for both the non-irradiated and irradiated samples according to its age are presented in Figure 4. A decrease in the values for both the irradiated and non-irradiated samples are within $3 \%$, while slightly lower changes can be noticed for the irradiated samples. Irradiation, therefore, does not have any significant effects on the bulk density of any of the investigated pastes.

Figure 5 shows the results of the non-destructive testing by the ultrasound pulse velocity method. The calculated values of the dynamic Young's modulus $E_{c u}$ show large differences between the individual types of cement pastes, however the relative differences between the irradiated and non-irradiated samples for each type are lower. The decrease in the values for the irradiated samples compared to the non-irradiated ones were, in the case of the reference cement paste, higher by $9 \%$, while for MF20, it was higher by $32 \%$. It should be noted that, in the case of MF20, the absolute decrease in the values was by $50 \%$ and $72 \%$ for non-irradiated and irradiated samples, respectively, which is a much higher drop than for the reference cement paste. Pastes with the superplasticizer Melflux 4930 even showed an increase in the case of the irradiated samples and the difference against to the drop in the values of the non-irradiated samples was by $11 \%$. The method, however, shows a high scatter of the results and a large measurement error. Therefore, the relative differences between the non-irradiated and the irradiated samples within $30 \%$ is not very significant. Generally, it can be stated that pastes containing $20 \%$ of the superplasticizer Melment F10 behaved worse than pastes containing $5 \%$ of the Melflux 4930, while the reference paste showed the best results.

Figures 6-8 present the obtained values of the impact echo method - the dynamic modulus of elasticity in the longitudinal direction, the transversal direction and the shear modulus, respectively. The changes in the obtained values over time are for both the non-irradiated and irradiated samples, i.e., before and after ageing, respectively, the irradiation is within the range of the measurement error. Moreover, there are even rises in the values of reference and M5 pastes due to the irradiation. In the case of the MF20 pastes, there are decreases in the values for both the non-irradiated and irradiated samples, the changes are within the measurement error, except for the dynamic modulus in the transversal direction.

The results of the destructive tests are presented in Figures 9 and 10. Irradiation of the samples had a positive effect on both the flexural (Figure 9) and the compressive strength (Figure 10). In the case of the flexural strength, the highest difference was recorded for 
the M5 pastes being $35 \%$, while the differences in the reference and MF20 pastes were within the test error. The highest absolute values were recorded for the pastes with the Melflux superplasticizer. Also, in the case of the compressive strength, the highest difference (almost by $30 \%$ ) was found for the M5 pastes, however, the highest absolute values were recorded for the reference pastes.

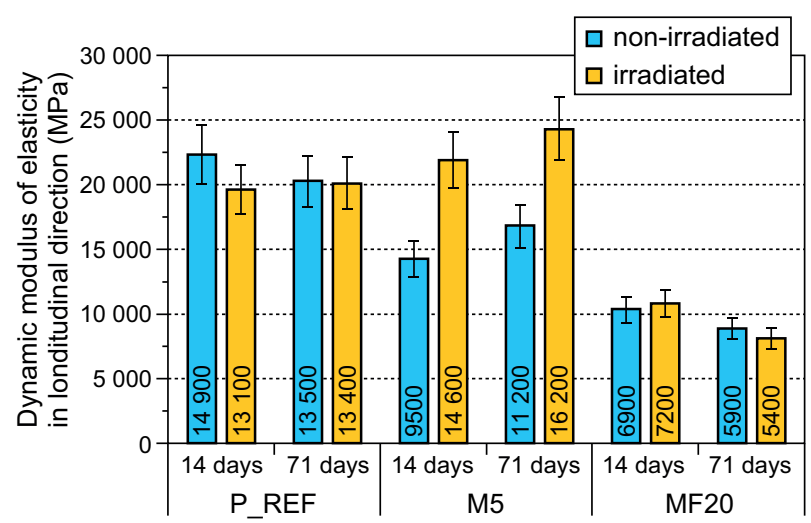

Figure 6. The dynamic modulus of elasticity in the longitudinal direction measured by the impact echo method before and after the irradiation compared to the non-irradiated samples.

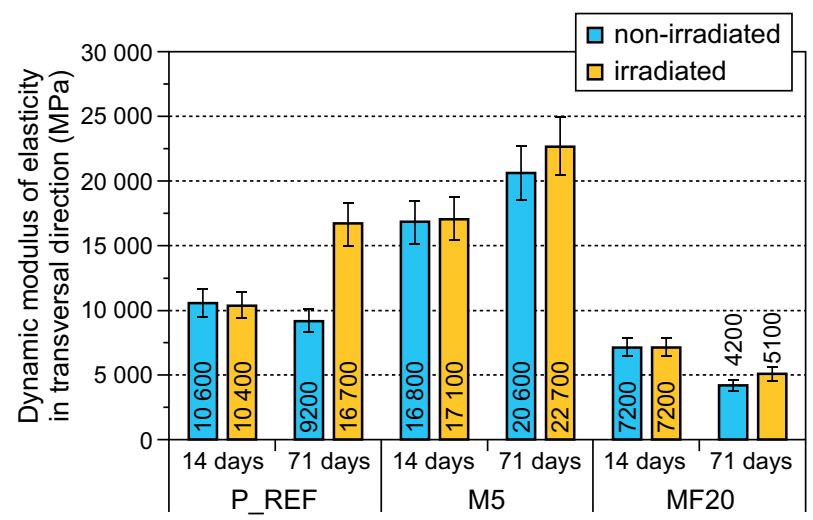

Figure 7. The dynamic modulus of elasticity in the transversal direction measured by the impact echo method before and after the irradiation compared to the non-irradiated samples.

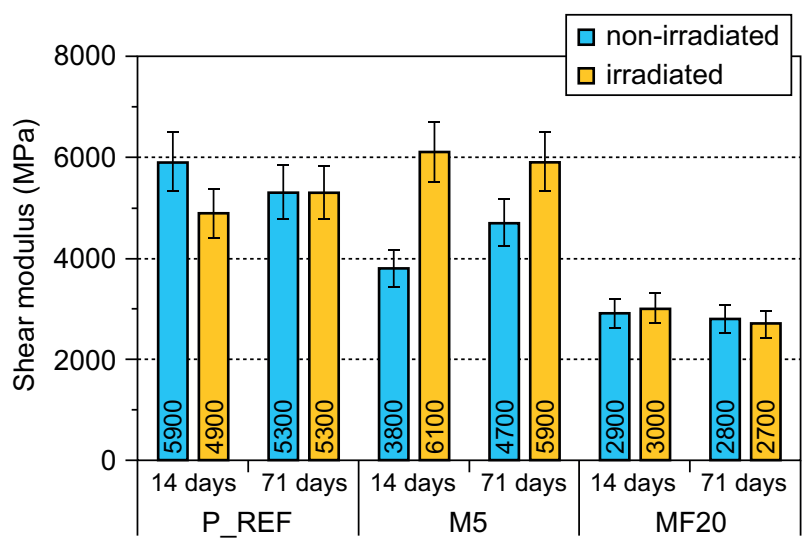

Figure 8 . The shear modulus measured by the impact echo method before and after the irradiation compared to the nonirradiated samples.
The positive differences in favour of the irradiated samples compared to non-irradiated ones were $17 \%$ and $9 \%$ for reference paste and MF20 paste, respectively.

\section{CONCLUSION}

The work focuses on the investigation of the properties of cement pastes incorporating high amounts of superplasticizers after irradiation by the gamma source Co-60. The cumulated radiation dose was up to $2.6 \mathrm{MGy}$. The results showed negligible effects of the irradiation on the bulk density of all the studied pastes. The values of the dynamic modulus of elasticity and the shear modulus obtained by non-destructive methods showed high scatter and, thus, the differences in the values are rather indicative. Generally, irradiation had a positive effect on the modulus of elasticity in the case of the pastes with Melflux 4930 (M5), while the reference paste and the paste with Melment F10 (MF20) were affected adversely. On the other hand, the destructive tests provably showed a positive effect of the irradiation on the mechanical properties of the studied cement pastes, as both the flexural and compressive strength were enhanced after

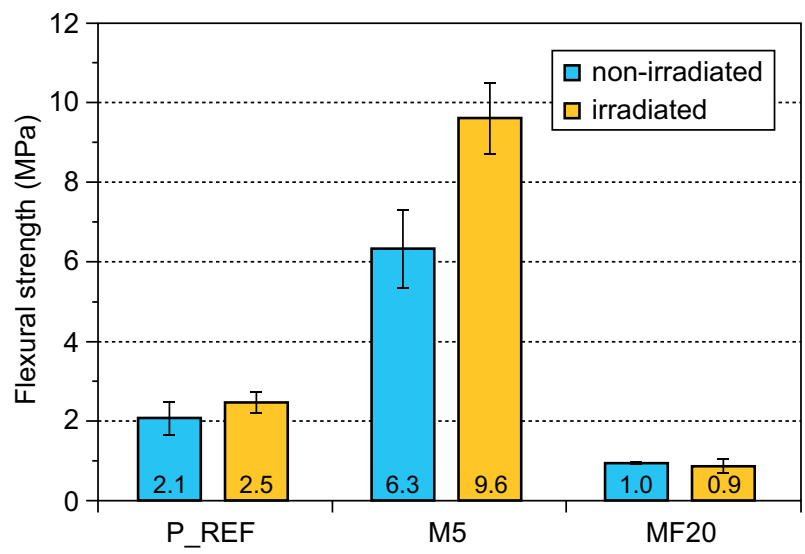

Figure 9. The flexural strength of the non-irradiated and irradiated samples.

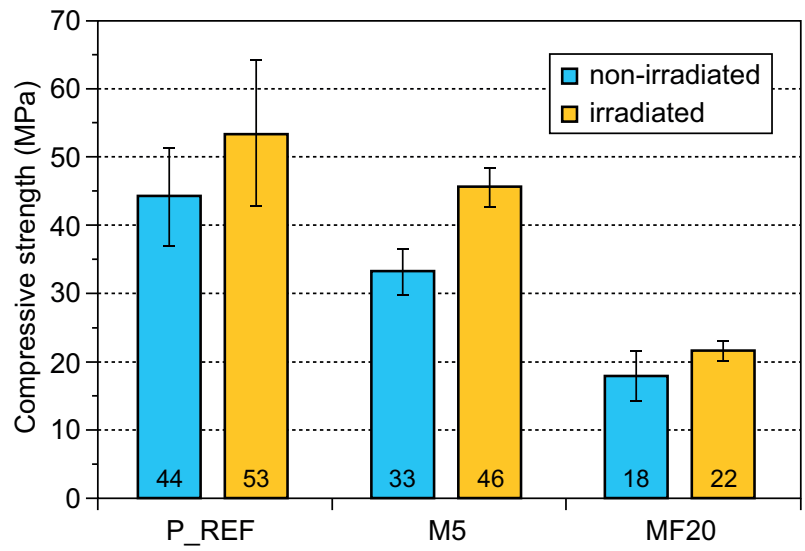

Figure 10. The compressive strength of the non-irradiated and irradiated samples. 
irradiation. The most pronounced effect was recorded for the M5 pastes. The recorded temperature changes inside the pastes were within $2^{\circ} \mathrm{C}$, being insignificant in the terms of a temperature gradient creation or the influence on other properties of the cement pastes.

\section{Acknowledgement}

The fabrication and testing of the prisms has been financially supported partially by the Czech Technical University in Prague - project no. SGS16/199/ OHK1/3T/11 and by the Czech Science Foundation, under project GA17-11635S.

The irradiation and related measurements of the temperature and the gamma irradiation dose has been funded by the Ministry of Education, Youth and Sport in the Czech Republic - LQ1603 Research for SUSEN under the projects CZ.1.05/2.1.00/03.0108 and CZ.02.1. 01/0.0/0.0/15 008/0000293.

\section{REFERENCES}

1. Mallants, D., Ochs, M., and Wang, L., Radionuclide and Metal Sorption on Cement and Concrete. Springer Verlag, DE.

2. McCulloch, C.E., Cements in Radioactive Waste Disposal: Some Mineralogical Considerations. Mineralogical Magazine, 49(351), 211-221, 1985.

3. Ochs, M., Colàs, E., Grivé, M., Olmeda, J., Campos, I., and Bruno, J., Reduction of radionuclide uptake in hydrated cement systems by organic complexing agents: Selection of reduction factors and speciation calculations. Svensk Kärnbränslehantering AB (SKB), Stockholm, Sweden.

4. ČSN 73 1371: Non-destructive testing of concrete-Method of ultrasonic pulse testing of concrete.

5. ČSN EN 196-1 Methods of testing cement - Part 1: Determination of strength. 2005.

6. ČSN EN 73 1372: Non-destructive testing of concrete Testing of concrete by resonance method on prisms. 2012 . 